\title{
LA EXPLORACIÓN VAGINAL DURANTE LA ASISTENCIA AL PARTO
}

\section{VAGINAL EXAMINATION IN MIDWIFERY}

\author{
Ballesteros Meseguer, Carmen \\ López Pay, María Dolores \\ Martínez Román, Concepción
}

Servicio de partos. Hospital Universitario Virgen de la Arrixaca. Murcia.

E-mail: carmenbm@um.es

\section{TÍTULO}

La exploración vaginal durante la asistencia al parto: un procedimiento basado en la evidencia científica.

\section{RESUMEN}

El tacto vaginal es un procedimiento que se utiliza con mucha frecuencia durante la asistencia a la mujer gestante tanto en el periodo del parto como durante el seguimiento del embarazo. La exploración vaginal, es una de las técnicas diagnósticas fundamentales en el comienzo y el progreso del parto. El objetivo de nuestro trabajo es desarrollar un protocolo, teniendo en cuenta las 
recomendaciones de la evidencia científica, y por otro lado reducir el riesgo de infección intraparto, materna y neonatal. Los beneficios que esperamos obtener con la elaboración de este protocolo son, por un lado, la mejora de la calidad de los cuidados enfermeros en la aplicación del tacto vaginal, y por otro lado la disminución de complicaciones durante la realización del mismo.

\section{PALABRAS CLAVE}

Examen ginecológico, tacto vaginal, matrona, mujer gestante.

\section{TITLE}

Vaginal examination in midwifery.

\section{ABSTRACT}

The pelvic exam is a procedure widely used for assisting pregnant women in both the delivery period and sometimes for the monitoring of pregnancy. Vaginal examination is one of the key diagnostic in the onset and progression of labor. The aim of our work is formalize this action to standardize the performance, taking into account the recommendations of the scientific evidence, and secondly to reduce the risk of intrapartum infection, maternal and neonatal mortality. The benefits expected from the development of this protocol on the one hand, improving the quality of nursing care in the application of vaginal examination, and secondly the reduction of complications during fulfillment.

\section{KEYWORDS}

Gynecological Examination, Vaginal Examinations, Midwifery, Pregnant Women

\section{INTRODUCCIÓN:}

El tacto vaginal es un procedimiento en la asistencia a la mujer embarazada durante el periodo de dilatación, en la fase de parto, y utilizado en 
medio hospitalario.

El número de tactos, el cuidado que se pone en su realización y, sobre todo, la duración del parto influyen en la incidencia de la infección materna y neonatal. 1,19

Diversos estudios demuestran que el tacto vaginal debe ser considerado como un factor de riesgo potencial de padecer una infección amniótica o puerperal, materna y neonatal. 2, 19

El tacto vaginal es siempre un tacto séptico, incluso realizándolo en las mejores condiciones asépticas, porque la vagina contiene bacterias, una flora que es inocua en la vagina misma, pero los dedos, por estériles que estén, arrastran bacterias hasta el orificio externo del cuello, y a través de él, hasta la cavidad uterina que tiene pocas defensas contra la infección. ${ }^{3,}{ }^{19}$. Por tanto sólo debe ser realizado por personal experto, con manos limpias y cubiertas por guantes estériles. En los hospitales universitarios el examen vaginal que puede ser llevado a cabo por un residente, a veces tendrá que ser repetido y confirmado por el supervisor. Esto sólo puede hacerse bajo el consentimiento de la mujer. Bajo ninguna circunstancia debe una mujer ser forzada a someterse a frecuentes y repetidos exámenes vaginales por las matronas o residentes 20 . El Objetivo del presente estudio es revisar la evidencia científica para estandarizar la actuación de la enfermera obstétrico-ginecológica (matrona) en la exploración vaginal, y por tanto, reducir el riesgo de infección intraparto, materna y neonatal. ${ }^{19}$

\section{DESCRIPCIÓN DEL PROCEDIMIENTO}

1. Revisar la Ha Clínica, evaluar el riesgo y la necesidad de realizar el procedimiento (Cat IC).

2. Informar a la mujer de la técnica a realizar y explicar las razones de la 
exploración y sus implicaciones. Obtener su consentimiento (Cat IC) ${ }^{4}$

3. Valorar si existe alergia al látex. Seleccionar guantes que no sean de látex si la mujer es alérgica.

4. Preparar el material y trasladarlo al lado de la mujer (CatIB).

5. Pedirle a la mujer que vacíe la vejiga antes del procedimiento si lo desea. Si es portadora de anestesia epidural, valorar la necesidad de sondaje vesical.

6. Favorecer un clima de confianza, seguridad e intimidad, respetando la privacidad, dignidad y comodidad durante la exploración (CatIC).

7. Colocar a la mujer en posición decúbito supino con los muslos flexionados y aducidos (posición ginecológica).

Aclaración: si la mujer lo desea se puede realizar en decúbito lateral con pierna superior flexionada y aducida, en posición genupectoral, de pie o en cuclillas.

8. Cubrir a la mujer con una sabana dejando acceso al periné.

9. Lavar con agua del grifo la región vulvoperineal. 15,16,17,18, 19

10. Realizar lavado de manos higiénico (Cat IA).

11. Colocar guantes estériles

12. Pedir a la mujer que relaje los muslos y las piernas.

Fundamento: La relajación reduce la tensión muscular y aumenta la comodidad.

13. Con los dedos pulgar y anular separar los labios mayores y menores para entreabrir el introito.

14. Insertar los dedos índice y medio bien lubricados de la mano dominante en la vagina hasta que toque el cérvix. Los dedos se introducen unidos y rígidos, deprimiendo el periné hacia abajo, reconociendo así el estado de la vagina, el relieve de los elevadores del ano y la integridad del periné. Se llega hasta contactar con el cuello del útero. 
15. Colocar la mano con la muñeca recta y el codo ligeramente hacia abajo.

Fundamento: Esta postura permite que el extremo de los dedos se dirija hacia el ombligo y lleguen al cérvix.

16. Si la mujer manifiesta estar molesta, escucharla y pedir disculpas.

Fundamento: Esto valida los sentimientos de la mujer y la ayuda a controlarse mejor.

17. Realizar la exploración vaginal durante y entre las contracciones.

Fundamento: la dilatación cervical, el borramiento y la estación o plano fetal se ven afectados por una contracción.

18. Palpar si en el cuello hay una apertura o depresión. Estimar el diámetro de la depresión para identificar el grado de dilatación.

El grado de dilatación cervical se mide en centímetros. El parto se inicia cuando es de 2-3 centímetros y llega a la dilatación completa cuando no se alcanza el cuello por delante de la presentación, que suele ser a los $10 \mathrm{~cm}$.

El grado de borramiento del cuello se mide en porcentaje sobre el resto del canal que permanece o directamente determinando la longitud en cm entre el orificio cervical interno y el externo.

19. Observar si se produce salida de líquido amniótico, color y olor.

Fundamento: determinar el estado de las membranas fetales y del líquido amniótico.

20. Palpar la presentación fetal. (porción del feto que entra primero en la pelvis) (ver anexos 1 y 2 )

Fundamento: proporciona información con respecto al descenso del feto y los movimientos cardinales.

21. Valorar el descenso fetal: valorar la altura de la presentación e identificar la posición de la fontanela posterior, menor o lamboidea.

Fundamento: proporciona información con respecto al descenso y la posición del feto.

22. Retirar suavemente los dedos. 
23. Realizar lavado de zona perineal con agua del grifo (Cat II).

24. Retirar empapador o cubrecama sucio y recoger material (Cat IA).

25. Colocar empapador limpio.

26. Colocar compresa higiénica ginecológica.

27. Cubrir con sabana limpia a la mujer.

28. Dejar a la mujer en una posición cómoda y adecuada.

29. Retirarse los guantes (Cat IA).

30. Realizar lavado de manos higiénico. (Cat IA).

31. Anotar en partograma (Cat II).

- Fecha

- Hora

- Longitud, dilatación, dureza, posición, del cérvix. (Tabla 1 Test de Bisop)

- Presentación fetal, altura de la presentación y posición. (Figura 1 Presentación fetal, Tabla 2 Variedades de posiciones de la cabeza fetal)

- Estado de la bolsa amniótica.

- Si existe dinámica uterina establecida.

Fundamento: el curso de la dilatación cervical depende de la efectividad de las contracciones uterinas, de la relación entre la pelvis y el feto y de la resistencia del canal blando del parto.

- Nombre de la matrona.

32. Explicar los hallazgos de forma comprensible y adecuada a sus necesidades (Cat IA $)^{4}$

33. Observaciones:

- Basándonos en la Teoría de la Enfermería Transcultural, tendremos en cuenta las preferencias de la mujer respecto a quién y de qué forma ha de llevarse a cabo el procedimiento. 
- No rasurar la zona porque puede causar infección e incomodidad. $(\text { Cat IA })^{5,19}$

- No reintroducir los dedos, en caso de duda volver a colocar un nuevo guante estéril.

Fundamento: aumenta el riesgo de infección por arrastre de gérmenes que colonizan la vagina.

El riesgo de infección se incrementa con el número de los tactos vaginales. Además, el número de tactos vaginales en el manejo de la primera etapa del parto tras la rotura prematura de membranas, es el factor independiente más importante para predecir una infección materna y/o neonatal. ${ }^{19}$

- Si al realizar el tacto vaginal advertimos la presencia de cordón, no extraer los dedos, rechazar la presentación fetal hacia arriba, para mantener la cabeza del feto fuera de la pelvis menor, solicitar ayuda médica urgente y colocar a la mujer en posición de Trendelenbur, en posición genupectoral o elevar la pelvis respecto al plano de la cabeza. ${ }^{21}$

- Si al realizar el tacto vaginal tocamos una superficie irregular a modo de vasos sanguíneos (vasa previa), no debemos manipular, por el riesgo de rotura, avisar al facultativo con carácter de urgencia. $^{22}$

- Un mismo tacto puede tener apreciaciones distintas por lo que el tacto, para ser mas objetivo en su evolución, debería ser realizado siempre por el mismo profesional dentro de su turno, salvo que precise valoración por facultativo. Así, se disminuye también el número de tactos innecesarios con el consiguiente aumento del riesgo de infección.

- El número de tactos será reducido al mínimo necesario para valorar con seguridad la evolución del parto (CAT IB), se recomienda su realización cada 2-4 horas o antes si el equipo obstétrico lo cree 
conveniente. Se practicará un tacto vaginal tras la amniorrexis, antes de la administración de analgesia epidural, tras la aparición de un registro cardiotocográfico patológico sin causa aparente o cuando la paciente presente deseos de realizar pujos. ${ }^{6}, 19$

Aclaración: la dinámica uterina consideraremos que es adecuada cuando permita el progreso satisfactorio de la dilatación cervical, sin repercusiones adversas para la madre o el feto, por tanto para su valoración deben conjugarse dos aspectos, registro de contracciones y dilatación cervical. ${ }^{7}$

\section{INDICACIONES:}

Pacientes gestantes, ingresadas en la unidad de dilatación durante la asistencia al parto, atendidas en el Hospital Universitario Virgen de la Arrixaca.

\section{CONTRAINDICACIONES:}

El tacto vaginal no se podrá realizar ante:

a) Placenta previa, sospechada o diagnosticada.

b) Hemorragia vaginal de origen desconocido.

c) Situación fetal transversa o presentaciones distintas a la cefálica.

\section{EVIDENCIA DISPONIBLE:}

El método empleado para identificar y revisar las evidencias científicas en las que se fundamenta este protocolo ha sido:

- Búsquedas en Sumarios de evidencia: Preevid (4 preguntas), Guías de salud. 
- Revisiones sistemáticas: Biblioteca Cochrane Plus, Centro Colaborador Instituto Joanna Briggs.

- Bases de datos: Pubmed.

- Legislación en BOE (Boletín oficial del estado).

- Las palabras clave utilizadas: Vaginal examinations. Vaginal examination labour. Chlorexidine vaginal.

- MeSH: vaginal exam, vaginal examination.

Las recomendaciones de evidencia científica (en nuestro hospital seguimos las del Instituto Joanna Brigg) y normas de proceso de los Centros para el control y prevención de enfermedades (CDC), van asociadas a las recomendaciones de procedimiento o a la técnica enlazándose con la referencia bibliográfica.

\begin{tabular}{|l|l|}
\hline Calidad de la evidencia & \multicolumn{1}{c|}{ Recomendación } \\
\hline BUENA & $\begin{array}{l}\text { Existe adecuada evidencia científica } \\
\text { Para recomendar o desaconsejar la } \\
\text { adopción de la tecnología. }\end{array}$ \\
\hline REGULAR & $\begin{array}{l}\text { Existe cierta evidencia científica para } \\
\text { recomendar o desaconsejar la la } \\
\text { adopción de la tecnología. }\end{array}$ \\
\hline BAJA & $\begin{array}{l}\text { Existe insuficiente evidencia científica } \\
\text { para recomendar o desaconsejar la } \\
\text { adopción de la tecnología. }\end{array}$ \\
\hline
\end{tabular}

La mejora en el desarrollo del trabajo enfermero vendrá determinado por el seguimiento de las recomendaciones de los CDC (Centros para el control y prevención de enfermedades) cuya finalidad es promover la salud y la calidad de vida mediante la prevención y el control de las enfermedades, las lesiones e incapacidades. Estas recomendaciones basadas en la evidencia de los datos científicos existentes, en los conceptos teóricos, en su aplicabilidad y en su 
impacto económico son agrupadas en las categorías siguientes según las recomendaciones de los CDC:

\begin{tabular}{|l|l|l|l|l|}
\hline \multicolumn{1}{|c|}{ Cat IA } & \multicolumn{1}{|c|}{ Cat IB } & \multicolumn{1}{c|}{ Cat IC } & \multicolumn{1}{c|}{ Cat II } & \multicolumn{1}{c|}{ NE } \\
\hline Normas & Normas & Requerida & Normas & Evidencia y \\
obligadas & obligadas & por & sugeridas. & consenso \\
con & evaluadas & regulaciones & Estudios & insuficiente \\
evidencia y & por del estado & clínicos y/o & \\
soporte & epidemiológi & \\
científico & $\begin{array}{l}\text { comité de } \\
\text { expertos }\end{array}$ & & cos & \\
\hline
\end{tabular}

Todos los estudios se clasifican según el nivel de la evidencia basándonos en el siguiente sistema de clasificación revisado.

\begin{tabular}{|l|l|}
\hline NIVEL I & $\begin{array}{l}\text { Evidencia obtenida de una revisión sistemática } \\
\text { de todos los ensayos clínicos con asignación } \\
\text { aleatoria relevante. }\end{array}$ \\
\hline NIVEL II & $\begin{array}{l}\text { Evidencia obtenida de al menos un ensayo clínico } \\
\text { con asignación aleatoria bien diseñado. }\end{array}$ \\
\hline NIVEL III.1 & $\begin{array}{l}\text { Evidencia obtenida de ensayos clínicos sin } \\
\text { asignación aleatoria bien diseñados. }\end{array}$ \\
\hline NIVEL III.2 & $\begin{array}{l}\text { Evidencia obtenida de estudios de cohortes o } \\
\text { casos y y controles bien diseñados, } \\
\text { preferiblemente de más de un centro o grupo de } \\
\text { investigación. }\end{array}$ \\
\hline NIVEL III.3 & $\begin{array}{l}\text { Evidencia obtenida de series temporales con o } \\
\text { sin intervención. Resultados importantes en } \\
\text { experimentos no controlados.| }\end{array}$ \\
\hline NIVEL IV & $\begin{array}{l}\text { Opinión de profesionales de reconocido prestigio, } \\
\text { basada en experiencia clínica, estudios } \\
\text { descriptivos o informes de comités de expertos. }\end{array}$ \\
\hline
\end{tabular}




\section{BIBLIOGRAFÍA}

1. González-Merlo. Obstetricia. $4^{a}$ edición. 1992. Edit. Masson. Barcelona.

2. DONAT COLOMER F. Enfermería maternal y ginecológica. Masson, Barcelona 2001.

3. Acién-Álvarez, P. Tratado de Obstetricia y Ginecología, Obstetricia. Alicante: Editorial Molloy, 1998.

4. Ley Básica reguladora de la autonomía del paciente y de derechos y obligaciones en materia de información y documentación clínica. Ley 41/2002 de 14 de noviembre. BOE, n0274, (15-11-2002).

5. Basevi V, Lavender T. Rasurado perineal sistemático en el ingreso a la sala de partos (Revisión Cochrane traducida). En: La Biblioteca Cochrane Plus, 2008 Número 4. Oxford: Update Software Ltd. Disponible en: http://www.update-software.com. (Traducida de The Cochrane Library, 2008 Issue 3. Chichester, UK: John Wiley \& Sons, Ltd.). Enfermería practice Bruner. Edit. Interamericana. 1981.

6. SEGO Documento de consenso asistencia al parto.2007.

7. López G., Comino R. Obstetricia y Ginecología. Edit. Ariel. 2004. Barcelona.

8. Fabre E. Manual de asistencia al embarazo normal. Edit. Ebrolibro. 1993. Zaragoza.

9. Jean Dickason Elizabeth. Enfermería Materno-Infantil Madrid España. Mosby/Doyma. 2a Edic. 1996.

10. Martínez Roche M.E., Pina Roche F., Gómez García C. Manual de procedimientos de Enfermería Materno-Infantil. Univ. de Murcia. 1994.

11. Neeson J. Consultor de Enfermería Obstétrica. Edit. Centrun. 1987.

12. Olds S., London M., Leadewig P.Enfermería Materno Infantil. 5a Edic. McGraw-Hill. Interamericana.

13. Romani F., Quispe J. Percepción sobre la experiencia del tacto vaginal durante el trabajo de parto. CIMEL 2007; 12(1): 11-15.

14. Vanrell J.A., et all. Manual de obstetricia y ginecología para pregraduados. Ediciones Científicas y técnicas. Masson. 1993. Barcelona.

15. Intrapartum care. Care of healthy women and their babies during 
childbirth. September 2007. NICE clinical guideline 55.

16. Stade B, Shah V, Ohlsson A. Clorhexidina vaginal durante el trabajo de parto para la prevención de la infección neonatal por estreptococo grupo B de comienzo temprano (Revisión Cochrane traducida). En: La Biblioteca Cochrane Plus, 2008 Número 4. Oxford: Update Software Ltd. Disponible en: http://www.update-software.com. (Traducida de The Cochrane Library, 2008 Issue 3. Chichester, UK: John Wiley \& Sons, Ltd.). Fecha de la modificación significativa más reciente: 18 de noviembre de 2003.

17. Lumbiganon $\mathrm{P}$, Thinkhamrop J, Thinkhamrop B, Tolosa JE. Clorhexidina vaginal durante el trabajo de parto para la prevención de infecciones maternas y neonatales (sin considerar las infecciones por estreptococo del grupo B y VIH) (Revisión Cochrane traducida). En: La Biblioteca Cochrane Plus, 2008 Número 4. Oxford: Update Software Ltd. Disponible en: http://www.update-software.com. (Traducida de The Cochrane Library, 2008 Issue 3. Chichester, UK: John Wiley \& Sons, Ltd.). Fecha de la modificación significativa más reciente: 07 de setiembre de 2003.

18. Saleem S, Rouse DJ, McClure EM, Zaidi A, Reza T, Yahya Y, Memon IA, Khan NH, Memon G, Soomro N, Pasha O, Wright LL, Moore J, Goldenberg RL. Chlorhexidine vaginal and infant wipes to reduce perinatal mortality and morbidity: a randomized controlled trial. Obstet Gynecol. 2010 Jun;115(6):1225-32.

19. Guía de Práctica Clínica sobre la Atención al Parto Normal. Ministerio de Sanidad y política social. 2010. Pág. 83-84.

20. Grupo de Trabajo técnico Organización Mundial de la Salud. Cuidados en el parto normal: Una guía práctica. Ginebra 1996.

21. VALENTI, Eduardo A. Guía de procidencia de cordón.Revista del Hospital Materno Infantil Ramón Sardá. 2008, vol. 27. Disponible en Internet: $\quad$ http://redalyc.uaemex.mx/src/inicio/ArtPdfRed.jsp? iCve=91211281005. ISSN 1514-9838.

22. Derbala Y, Grochal F, Jeanty P. Vasa previa. J Prenat Med. 2007; $1: 2-13$. 
Tabla 1: Test de Bisop. (1964)

\begin{tabular}{|l|l|l|l|l|}
\hline \multicolumn{5}{|c|}{ PUNTUACIÓN } \\
\cline { 2 - 5 } & 0 & 1 & 2 & 3 \\
\hline $\begin{array}{l}\text { Longitud } \\
\text { cérvix }(\mathrm{cm})\end{array}$ & $+2 \mathrm{~cm}$ & 2 & 1 & 0.5 \\
\hline Consistencia & Duro & Semiduro & Blando & - \\
\hline $\begin{array}{l}\text { Dilatación } \\
\text { cm) }\end{array}$ & 0 & $1-2$ & $3-4$ & +4 \\
\hline Posición & Posterior & Intermedia & central & - \\
\hline ESTACION & SES-I & I-II & II-III & III-IV \\
\hline
\end{tabular}

Tabla 2: VARIEDADES DE POSICIONES DE LA CABEZA FETAL (Fuente: Vanrell 1993)

\begin{tabular}{|c|c|c|}
\hline Presentaciones & Variedad de posición & siglas \\
\hline $\begin{array}{l}\text { Cefálica } \\
\text { vértice }\end{array}$ & $\begin{array}{l}\text { Occipitopúbica } \\
\text { Occipitoilíaca izquierda anterior } \\
\text { Occipitoilíaca izquierda transversa } \\
\text { Occipitoilíaca izquierda posterior } \\
\text { Occipitosacra } \\
\text { Occipitoilíaca derecha posterior } \\
\text { Occipitoilíaca derecha transversa } \\
\text { Occipitoilíaca derecha anterior }\end{array}$ & $\begin{array}{l}\text { OP } \\
\text { OIIA } \\
\text { OIIT } \\
\text { OIIP } \\
\text { OS } \\
\text { OIDP } \\
\text { OIDT } \\
\text { OIDA }\end{array}$ \\
\hline $\begin{array}{l}\text { Cefálica } \\
\text { bregma }\end{array}$ & $\begin{array}{l}\text { Frontopúbica } \\
\text { Frontoilíaca izquierda anterior } \\
\text { Frontoilíaca izquierda transversa } \\
\text { Frontoiliaca izquierda posterior } \\
\text { Frontosacra } \\
\text { Frontoilíaca derecha anterior } \\
\text { Frontoilíaca derecha transversa } \\
\text { Frontoilíaca derecha posterior }\end{array}$ & $\begin{array}{l}\text { FP } \\
\text { FIIA } \\
\text { FIIT } \\
\text { FIIP } \\
\text { FS } \\
\text { FIDA } \\
\text { FIDT } \\
\text { FIDP }\end{array}$ \\
\hline $\begin{array}{l}\text { Cefálica } \\
\text { frente }\end{array}$ & $\begin{array}{l}\text { Nasopúbica } \\
\text { Nasoilíaca izquierda anterior } \\
\text { Nasoilíaca izquierda transversa } \\
\text { Nasoilíaca izquierda posterior } \\
\text { Nasosacra } \\
\text { Nasoilíaca derecha anterior } \\
\text { Nasoilíaca derecha transversa } \\
\text { Nasoilíaca derecha posterior }\end{array}$ & $\begin{array}{l}\text { NP } \\
\text { NIIA } \\
\text { NIIT } \\
\text { NIIP } \\
\text { NS } \\
\text { NIDA } \\
\text { NIDT } \\
\text { NIDP }\end{array}$ \\
\hline $\begin{array}{l}\text { Cefálica } \\
\text { cara }\end{array}$ & $\begin{array}{l}\text { Mentopúbica } \\
\text { Mentoilíaca izquierda anterior } \\
\text { Mentoilíaca izquierda transversa } \\
\text { Mentoilíaca izquierda posterior } \\
\text { Mentosacra } \\
\text { Mentoilíaca derecha anterior } \\
\text { Mentoilíaca derecha transversa } \\
\text { Mentoilíaca derecha posterior }\end{array}$ & $\begin{array}{l}\text { MP } \\
\text { MIIA } \\
\text { MIIT } \\
\text { MIIP } \\
\text { MS } \\
\text { MIDA } \\
\text { MIDT } \\
\text { MIDP }\end{array}$ \\
\hline Nalgas & $\begin{array}{l}\text { Sacropúbica } \\
\text { Sacroilíaca izquierda anterior } \\
\text { Sacroiliaca izquierda transversa } \\
\text { Sacroiliaca izquierda posterior } \\
\text { Sacrosacra } \\
\text { Sacroilíaca derecha anterior } \\
\text { Sacroiliaca derecha transversa } \\
\text { Sacroiliaca derecha posterior }\end{array}$ & $\begin{array}{l}\text { SP } \\
\text { SIIA } \\
\text { SIIT } \\
\text { SIIP } \\
\text { SS } \\
\text { SIDA } \\
\text { SIDT } \\
\text { SIDP }\end{array}$ \\
\hline
\end{tabular}


Figura 1: PRESENTACION FETAL (Fuente: Manual Merck)

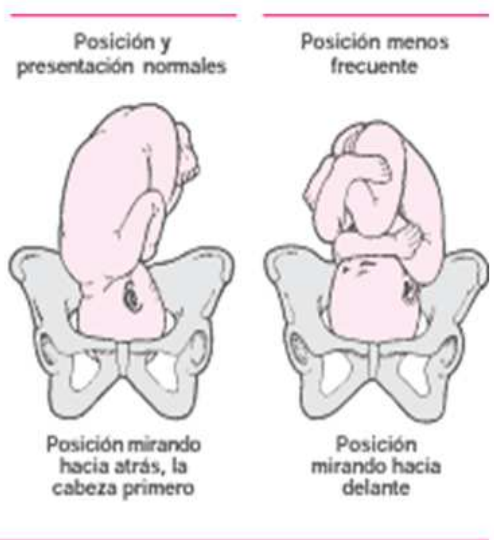

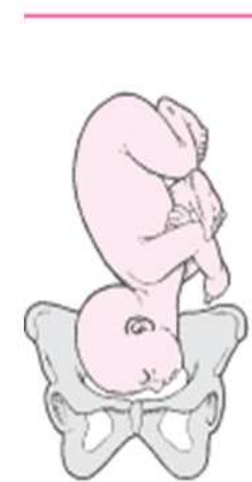

Cara

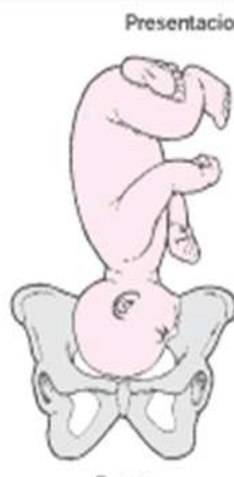

Frente

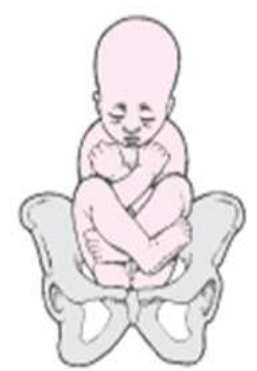

Nalgas

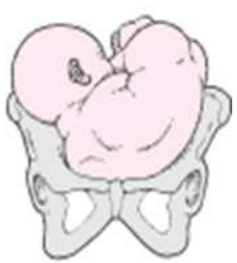

Hombro 